\title{
Prognostic indices predictive of short term survival in breast carcinoma in the Sri Lankan setting
}

\author{
H. D. Wijesinghe ${ }^{1}$, P. Thuvarakan ${ }^{1}$, G. K. Wijesinghe ${ }^{1}$, A. Samarasekera ${ }^{2}$ and \\ M. D. S. Lokuhetty ${ }^{1}$
}

Department of Pathology, Faculty of Medicine, University of Colombo, Sri Lanka ${ }^{1}$

National Hospital of Sri Lanka

DOI: http://doi.org/10.4038/jdp.v11i2.7721

\begin{abstract}
Introduction
There are many established and novel prognostic indices described for breast carcinoma (BCa). We aimed to study prognostic indices for $\mathrm{BCa}$ derived from routine clinical, histopathological and immunohistochemical data in the local setting and to evaluate their impact on short-term overall survival(OS) and disease free survival(DFS).
\end{abstract}

\section{Methodology}

Bidirectional cohort study included 174 BCa patients at National Hospital of Sri Lanka, from 2012-2014, excluding post-neo-adjuvant chemotherapy cases. Clinical details, tumour size and nodal status were obtained from histopathology records. Histopathological parameters, oestrogen and progesterone receptors and HER2 status were reviewed. Molecular subtype, nodal ratio (NR=number of positive nodes/total number retrieved) and Nottingham prognostic index (NPI) were

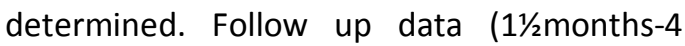
years) were obtained. Data was analyzed by life tables and Cox regression using SPSS 16.0.

\section{Results}

Mean age was 56.5 years (29-87years) and mean follow-up duration was 27.3 months(SD=8.68). Of the study group $148(85.1 \%)$ remained disease free, 10(5.7\%)survived with metastasis, $4(2.3 \%)$ survived with recurrences and 12(6.9\%) succumbed. Duration of breast lump $<3$ months (OS- $p=0.023$,DFS- $p=0.025$ ), NR $<0.5$
(OS-p<0.005, DFS-p<0.001), tumour stage (OS$p<0.001$,DFS- $p<0.001$ ), nodal stage (OS$p=0.013$, DFS $-p=0.015$ ) and TNM stage (OS$p=0.029$,DFS- $p=0.004$ ) were predictive of both OS and DFS on Wilcoxon test. Tumour stage was predictive of both OS $(p=0.028)$ and DFS $(p=0.008)$ on multivariate Cox regression analysis. Lymphoid infiltrate (LI) at tumour periphery was predictive of OS $(p=0.045)$. Age, other histopathological features, hormone/HER2 status, molecular subtype and NPI showed no significant effect on survival.

\section{Discussion and conclusion}

Stage, nodal ratio and lymphoid infiltrate were predictive of short-term survival similar to other studies. Exclusion of advanced cases possibly contributed to lack of significance of other prognostic factors. Breast lump duration, tumour, nodal and TNM stages and nodal ratio were predictive of both overall survival and disease free survival, with tumour stage being predictive on both univariate and multivariate analysis. Lymphoid infiltrate at tumour periphery was predictive of overall survival. 